\title{
Tratamiento de la Mucositis Oral en Pacientes Oncológicos. Revisión de la Literatura y Experiencia en el Hospital General de México
}

\author{
Management of Oral Mucositis in Patients with Cancer. Review \\ of Literature and Experience in the General Hospital of Mexico
}

\begin{abstract}
María Verónica Cuevas-González; Enrique Echevarría-y-Pérez ${ }^{* * * * *}$; Celia Minerva Díaz-Aguirre ${ }^{* * *} \&$ Juan Carlos Cuevas-González ${ }^{* * *}$
\end{abstract}

CUEVAS-GONZÁLEZ, M. V.; ECHEVARRÍA-Y- PÉREZ, E.; DÍAZ-AGUIRRE. C. M. \& CUEVAS-GONZÁLEZ, J. C. Tratamiento de la mucositis oral en pacientes oncológicos. Revisión de la literatura y experiencia en el Hospital General de México. Int. J. Odontostomat., 9(2):289-294, 2015.

RESUMEN: La mucositis es la reacción secundaria a la quimioterapia y/o radioterapia, existen múltiples opciones terapéuticas para tratar esta complicación, no se cuenta con un tratamiento definido por lo que el objetivo de esta revisión es el de agrupar las distintas maneras de abordar la mucositis oral y su efecto así como compartir la experiencia que se tiene en el Hospital General de México. Dentro de los tratamientos más utilizados se encuentra el empleo de fármacos protectores de mucosa, crioterapia, factores de crecimiento entre otros, el efecto deseado es retrasar la aparición de la lesión así como disminuir la severidad de las mismas, no todas las opciones se encuentran disponibles para el personal médico por lo que se deben de conocer las distintas opciones terapéuticas y la solidez científica con la que cuentan.

PALABRAS CLAVE: Hospital General de México, mucositis oral, pacientes oncológicos, revisión de la literatura.

\section{INTRODUCCIÓN}

La mucositis es la reacción secundaria a la quimioterapia y/o radioterapia que se caracteriza por la presencia de áreas eritematosas y lesiones ulcerativas en la mucosa oral, ocasionando dolor y limitaciones en la alimentación, siendo esta uno de los efectos secundarios más comunes dentro del tratamiento oncológico (Yarom et al., 2013).

Alrededor del $40 \%$ de los pacientes bajo tratamiento de quimioterapia desarrollan mucositis, esto dependiendo del tipo de agente quimioterapéutico empleado. En pacientes con tratamiento de radioterapia en la región de cabeza y cuello más del $90 \%$ puede presentar esta patología (Lalla et al., 2011). Como se muestra en la Tabla I, existen diversas clasificaciones para la mucositis, las que más destacan son la de la OMS y la de NCl (Puyal et al., 2003; Molina Giraldo \& Estupiñán Guzmán, 2010).
El objetivo de esta revisión es el de agrupar las múltiples opciones terapéuticas propuestas para la mucositis oral y su efecto, esto debido a que aun no se cuenta con un tratamiento que prevenga la aparición de dicha lesión, sin embargo la literatura maneja distintas maneras de abordar la mucositis para disminuir su severidad, que van desde opciones naturales, como la miel real o el propóleo hasta la aplicación de láser, agentes citoprotectores, anestésicos locales entre otros.

Fármacos protectores de mucosa. El empleo de fármacos citoprotectores se utilizan con frecuencia debido a su bajo costo y su facilidad al utilizarlos en forma de colutorio, en donde se combinan con anestésicos y/o antihistamínicos, los cuales forman una capa protectora sobre la mucosa e incrementan la síntesis de las prostaglandinas E2 favoreciendo la repa-

\footnotetext{
Alumna de la Maestría en Ciencias Estomatológicas, Facultad de Odontología, Universidad Juárez del Estado de Durango, Durango, México. ** Profesor de la Facultad de Odontología (DEPel), Universidad Nacional Autónoma de México, México D.F., México.

*** Profesora titular de la Especialidad en Prótesis Maxilofacial, Hospital General de México, México D.F., México.

***t Profesor investigador, Facultad de Odontología, Universidad Juárez del Estado de Durango, Durango, México.
} 
Tabla I. Clasificaciones más utilizadas de la mucositis (Puyal et al., 2003; Molina Giraldo \& Estupiñán Guzmán, 2010).

\begin{tabular}{lll}
\hline OMS & Grado & Característica \\
& 0 & Sin lesión. \\
& 1 & Eritema generalizado, mucosa rosada no dolorosa con abundante saliva. \\
& 2 & $\begin{array}{l}\text { Eritema, ulceras poco extensas, se mantiene deglución de sólidos. } \\
\text { Ulceras extensas, encías edematosas, saliva espesa, se mantiene la } \\
\text { capacidad de deglutir líquidos, dolor, dificultad para hablar. } \\
\text { Ulceras muy extensas, encías sangrantes, infecciones, no hay saliva, es } \\
\text { imposible deglutir, dolor muy intenso }\end{array}$ \\
& 3 & No mucositis. \\
Funcional y & 0 & Capaz de comer solidos. \\
Sintomática & 1 & Requiere dieta liquida. \\
& 2 & La alimentación no es posible. \\
& 3 & Síntomas asociados con consecuencias que ponen en peligro la vida. \\
Clínica & 4 & Eritema de la mucosa. \\
& 0 & Ulceración en parches o pseudomembranas. \\
& 1 & Ulceraciones confluentes o pseudomembranas. \\
& 2 & Necrosis tisular.
\end{tabular}

ración tisular, otra característica es que poseen propiedades anti-inflamatorias, analgésicas y antimicrobianas (Tejada Domínguez \& Ruiz Domínguez, 2010; Lalla et al., 2014).

El uso de la Benzidamina es el fármaco que cuenta con mayor soporte en la literatura sobre su eficacia ya que en diversos estudios demuestran que disminuye la severidad de la lesión al igual que la sintomatología dolorosa (Roopashri et al., 2011; Sheibani et al., 2015), esta evidencia se basa en estudios realizados en pacientes bajo tratamiento de radioterapia, la evidencia en pacientes con tratamiento concomitante de radioterapia y quimioterapia no es contundente (Nicolatou-Galitis et al., 2013). En cuanto al empleo de misoprostol, en ensayos clínicos realizados en pacientes con tratamiento de quimioterapia así como revisiones de la literatura no muestran diferencias en cuanto a la disminución del dolor y severidad de la lesión (Lalla et al., 2012).

La utilización de enjuagues a base de fosfato de calcio o clorhexidina son ampliamente utilizados, a pesar de que no hay suficientes estudios clínicos que detallen su efectividad en el manejo de la mucositis. (Campos et al., 2014). Se encuentran múltiples fórmulas para la preparación de la solución filadelfia, sus principales componentes son antihistamínicos, protectores de la mucosa, antifúngicos, anestésicos entre otros, su efecto se enfoca en la disminución de la severidad de las lesiones, disminución en la intensidad del dolor, mejorando la ingesta de alimentos del paciente (Mínguez \& De Andrés, 2005). En el uso de soluciones magistrales en pacientes pediátricos se han comparado el uso del hidróxido de aluminio con lidocaína y sucrafalto de aluminio con lidocaína así como lidocaína en gel, donde no se mostraron diferencias en la disminución de la severidad de la lesión (Barbosa Liz et al., 2009).

Crioterapia. La crioterapia en un método alternativo que al provocar vasoconstricción evita que el medicamento llegue a la mucosa oral causando daño, además de generar una sensación de adormecimiento (Alonso Castell et al., 2001). Revisiones sistemáticas realizadas acerca del uso de la crioterapia concluyen que es eficaz al reducir el desarrollo de la lesión así como disminuir la sintomatología dolorosa en pacientes en tratamiento con 5FU o melfalan (Svanberg et al., 2010; Peterson et al., 2013).

Ensayos clínicos donde se compara la crioterapia con el factor de crecimiento se muestra que la primera es mejor en la prevención de la mucositis, pero no en la disminución de la severidad una vez que se presenta (Kanuga, 2013). Este tipo de tratamiento tiene un enfoque hacia pacientes que se encuentran medicados con 5-FU o bencidamina, faltan más estudios con otro tipo de agentes quimioterapéuticos. 
Factores de crecimiento. El factor de crecimiento epidérmico es una opción terapéutica empleada por sus propiedades de ayudar a mantener la homeostasia tisular al regular la proliferación celular y promover la angiogénesis (Hong et al., 2009). Estudios a doble ciego demuestran que el uso de factor de crecimiento epidérmico a dosis de $50 \mathrm{ug} /$ $\mathrm{ml}$ es eficaz en el tratamiento de la mucositis (Wu et al., 2009).

El uso del factor de crecimiento queratinocitico también ha mostrado tener buenos resultados en pacientes sometidos a trasplante de células hematopoyéticas (Sonis, 2010), pero al igual que el anterior se requieren más estudios que sustenten su uso.

Terapia láser. El láser de baja potencia es utilizado debido a la bio-estimulación del tejido y a la acción analgésica y antiinflamatoria (Oberoi et al., 2014), se han publicado una serie de meta-análisis acerca de su uso reportando que la utilización del láser de baja intensidad es eficaz favoreciendo la prevención de la mucositis (Figueiredo et al., 2013). Estudios comparativos acerca del uso del láser y fármacos protectores de mucosa demuestran que la terapia láser es más efectiva al controlar el dolor y retrasar la evolución de la mucositis en pacientes con quimioterapia y radioterapia (Lima et al., 2010), el grueso de la literatura concuerda que se necesitan estudios con una mayor población.

Higiene oral. La higiene oral deficiente algunos autores la consideran como un factor desencadenante de la mucositis oral avanzada, estudios donde se evalúan las condiciones de higiene oral antes y durante el tratamiento de trasplante de células hematopoyéticas reportaron que el índice de placa bacteriana y la enfermedad periodontal son factores determinantes que intervienen en la severidad de la mucositis (Coracin et al., 2013; Hejna et al., 2001).

\section{Terapia con agentes naturales}

Aloe vera. El aloe vera, se ha utilizado desde la antigüedad para el tratamiento de heridas al optimizar su cicatrización (Ferraro, 2009), en el tratamiento de la mucositis oral se realizó un ensayo clínico comparando la eficacia del aloe vera con la Benzidamina en 26 pacientes bajo tratamiento de radioterapia en el cual no se mostraron diferencias significativas en cuanto a la disminución o al retraso en la aparición de la mucositis (Sahebjamee et al., 2015).

Miel de abeja. En estudios comparativos donde utilizaron miel y soluciones magistrales a base de Benzidamina y nistatina, se muestra que en estadios iniciales de la mucositis los enjuagues a base de miel acortan el tiempo de recuperación (Erdem \& Güngörmüs, 2014), con la limitante de que en estudios a doble ciego no se mostraron diferencias significativas en cuanto a la mejoría de la lesión (Bardy et al., 2012; Hawley et al., 2014). Otros autores al comparar el uso de la miel con el de la Benzidamina reportaron que la miel retarda la evolución de la lesión y evita el desarrollo de ésta a estadios avanzados en comparación con el grupo que uso Benzidamina (Jayachandran \& Balaji, 2012). La miel de abeja por sus propiedades antiinflamatorias, antibióticas y antioxidantes es una opción en el tratamiento de la mucositis, sin embargo se necesitan estudios más sólidos metodológicamente que confirmen dichos beneficios (Jayachandran \& Balaji).

En el Hospital General de México el servicio de prótesis maxilofacial, se encarga de realizar la valoración oral a todos los pacientes oncológicos antes de iniciar el tratamiento oncológico, en el cual se eliminan focos de infección y se les brindan indicaciones de higiene, esto se ha visto que aminora la severidad de la mucositis. Las características clínicas que presentan los pacientes con mucositis en la cavidad oral en nuestro servicio son eritema, presencia de múltiples úlceras en la mucosa las cuales presentan un tamaño que oscila de unos cuantos milímetros a varios centímetros y dolor que impide la alimentación (Fig. 1).

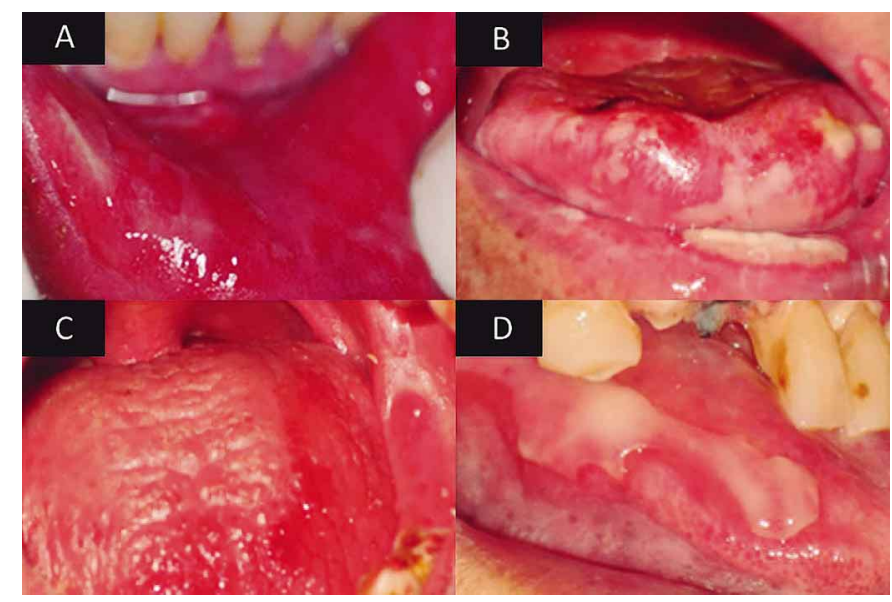

Fig. 1. Pacientes que acudieron al servicio de prótesis maxilofacial que estaban bajo tratamiento oncológico y desarrollaron algún grado de mucositis, en distintas zonas topográficas de la cavidad oral. 
CUEVAS-GONZÁLEZ, M. V.; ECHEVARRÍA-Y- PÉREZ, E.; DÍAZ-AGUIRRE. C. M. \& CUEVAS-GonZÁLEZ, J. C. Tratamiento de la mucositis oral en pacientes oncológicos. Revisión de la literatura y experiencia en el Hospital General de México. Int. J. Odontostomat., 9(2):289-294, 2015.

Cuando se presentan las lesiones, los métodos que se utilizan para tratar este padecimiento es mediante soluciones magistrales a base de caolín pectina, difenhidramina y anestésicos, los pacientes refieren una marcada disminución en el dolor, permitiéndoles la ingesta de alimentos, su periodo de cicatrización oscila entre los 7 y 10 días, tiempo que concuerda a lo reportado en la literatura debido al recambio epitelial en la mucosa oral. Este tratamiento va de la mano con una dieta libre de irritantes, una higiene oral con cepillo blando y libre de pasta dental, en caso que al paciente no le sea tolerable.

Los resultados que se obtienen en el Hospital General de México al tratamiento de la mucositis son muy parecidos a los descritos en la literatura en general, en donde la disminución de la sintomatología es el principal efecto. En nuestro hospital, en el servicio de prótesis maxilofacial se atienden pacientes de escasos recursos económicos por lo que no contamos con experiencia en otro tipo de tratamientos como terapia láser o uso de factores de crecimiento debido al costo que representan, y las terapias con tratamientos naturales debido a la falta de evidencia en población mexicana las limitamos y no las usamos de manera cotidiana. Por lo que proponemos realizar estudios donde se compare la efectividad de los métodos antes descritos para fundamentar el efecto.

\section{CONCLUSIONES}

Elegir un tratamiento para aminorar la sintomatología de la mucositis depende de la complejidad de las lesiones así como del recurso económico con el que se cuente. Apoyamos el uso de soluciones magistrales en concomitancia con una buena higiene y una dieta restringida en alimentos irritantes como una opción de tratamiento accesible y eficaz, debido al bajo costo, practicidad y efectividad.

CUEVAS-GONZÁlEZ, M. V.; ECHEVARRÍA-Y- PÉREZ, E.; DÍAZ-AGUIRRE. C. M. \& CUEVAS-GONZÁLEZ, J. C. Management of oral mucositis in patients with cancer. Review of literature and experience at the General Hospital of Mexico. Int. J. Odontostomat., 9(2):289-294, 2015.

ABSTRACT:Mucositis is the secondary reaction to chemotherapy and / or radiotherapy, there are multiple therapeutic alternatives to treat this complication, there doesn't exist a specific therapy so the aim of this review is to coordinate the various ways of treating oral mucositis and its effect and to share the experience at the General Hospital of Mexico. Within the most widely used treatments is the use of mucosal protective drugs, cryotherapy, growth factors, among others, the main effect is to delay the onset of injury and decrease the severity of them, not all options are available for medical staff so they must know the different Iptherapeutic alternatives and the scientific soundness for each option.

KEY WORDS: General Hospital of Mexico, oral mucositis, patients with cancer, review of literature.

\section{REFERENCIAS BIBLIOGRÁFICAS}

Alonso Castell, P.; Basté Dencàs, M. A.; Creus Viles, M.; Del Pino Gaya, B.; Gómez Blasco, C.; Gómez Gener, A.; Gorgas Torner, M. Q.; Muro Perea, N.; Pellicer Jacomet, M. A.; Sotoca Momblona, J. M.; Trullàs Altisen, M. \& Vallés Fernández, R. Prevención y tratamiento de la mucositis en el paciente onco-hematológico. Farm. Hosp., 25(3):139-49, 2001.

Barbosa Liz, D. M.; Bernal de Jaramillo, L. V.; Gallego González, C. \& Sierra Sánchez, M. E. Comparación de los efectos de tres enjuagues en el manejo de la mucositis oral secundaria al tratamiento de leucemia linfoblástica aguda en niños. Rev. Fac. Odontol. Univ. Antioq., 20(2):179-90, 2009.

Bardy, J.; Molassiotis, A.; Ryder, W. D.; Mais, K.; Sykes, A.;
Yap, B.; Lee, L.; Kaczmarski, E. \& Slevin, N. A doubleblind, placebo-controlled, randomised trial of active manuka honey and standard oral care for radiationinduced oral mucositis. Br. J. Oral Maxillofac. Surg., 50(3):221-6, 2012.

Campos, M. I.; Campos, C. N.; Aarestrup, F. M. \& Aarestrup, B. J. Oral mucositis in cancer treatment: Natural history, prevention and treatment. Mol. Clin. Oncol., 2(3):337-40, 2014.

Coracin, F. L.; Santos, P. S.; Gallottini, M. H.; Saboya, R.; Musqueira, P. T.; Barban, A.; Chamone, Dde. A.; Dulley, F. L. \& Nunes, F. D. Oral health as a predictive factor for oral mucositis. Clinics (Sao Paulo), 68(6):792-6, 2013. 
CUEVAS-GONZÁLEZ, M. V.; ECHEVARRÍA-Y- PÉREZ, E.; DíAZ-AGUIRRE. C. M. \& CUEVAS-GonZÁLEZ, J. C. Tratamiento de la mucositis oral en pacientes oncológicos. Revisión de la literatura y experiencia en el Hospital General de México. Int. J. Odontostomat., 9(2):289-294, 2015.

Erdem, O. \& Güngörmüs, Z. The effect of royal jelly on oral mucositis in patients undergoing radiotherapy and chemotherapy. Holist. Nurs. Pract., 28(4):242-6, 2014.

Ferraro, G. M. Revisión de la aloe vera (Barbadensis Miller) en la dermatología actual. Rev. Argent. Dermatol., 90(4):218-23, 2009.

Figueiredo, A. L.; Lins, L.; Cattony, A. C. \& Falcão, A. F. Laser therapy in the control of oral mucositis: a meta-analysis. Rev. Assoc. Med. Bras., 59(5):467-74, 2013.

Hawley, P.; Hovan, A.; McGahan, C. E. \& Saunders, D. A randomized placebo-controlled trial of manuka honey for radiation-induced oral mucositis. Support Care Cancer, 22(3):751-61, 2014.

Hejna, M.; Köstler, W. J.; Raderer, M.; Steger, G. G.; Brodowicz, T.; Scheithauer, W.; Wiltschke, C. \& Zielinski, C. C. Decrease of duration and symptoms in chemotherapy-induced oral mucositis by topical GMCSF: results of a prospective randomised trial. Eur. J. Cancer, 37(16):1994-2002, 2001.

Hong, J. P.; Lee, S. W.; Song, S. Y.; Ahn, S. D.; Shin, S. S.; Choi, E. K. \& Kim, J. H. Recombinant human epidermal growth factor treatment of radiation-induced severe oral mucositis in patients with head and neck malignancies. Eur. J. Cancer Care (Engl.), 18(6):636-41, 2009.

Jayachandran, S. \& Balaji, N. Evaluating the effectiveness of topical application of natural honey and benzydamine hydrochloride in the management of radiation mucositis. Indian J. Palliat. Care, 18(3):190-5, 2012.

Kanuga, S. Cryotherapy and keratinocyte growth factor may be beneficial in preventing oral mucositis in patients with cancer, and sucralfate is effective in reducing its severity. J. Am. Dent. Assoc., 144(8):928-9, 2013.

Lalla, R. V.; Bowen, J.; Barasch, A.; Elting, L.; Epstein, J.; Keefe, D. M.; McGuire, D. B.; Migliorati, C.; NicolatouGalitis, O.; Peterson, D. E.; Raber-Durlacher, J. E.; Sonis, S. T.; Elad, S. \& Mucositis Guidelines Leadership Group of the Multinational Association of Supportive Care in Cancer and International Society of Oral Oncology (MASCC/ISOO). MASCC/ISOO clinical practice guidelines for the management of mucositis secondary to cancer therapy. Cancer, 120(10):1453-61, 2014.

Lalla, R. V.; Brennan, M. \& Schubert, M. Oral complications of cancer therapy. In: Yagiela, J. A.; Dowd, F. J. \& Johnson, B. S. (Eds.). Pharmacology and Therapeutics for Dentistry. 6th ed. St. Louis, Mosby Elsevier, 2011.

Lalla, R. V.; Gordon, G. B.; Schubert, M.; Silverman, S. Jr.; Hutten, M.; Sonis, S. T.; LeVeque, F. \& Peterson, D. E. A randomized, double-blind, placebo-controlled trial of misoprostol for oral mucositis secondary to high- dose chemotherapy. Support Care Cancer, 20(8):1797-804, 2012.

Lima, A. G.; Antequera, R.; Peres, M. P.; Snitcosky, I. M.; Federico, M. H. \& Villar, R. C. Efficacy of low-level laser therapy and aluminum hydroxide in patients with chemotherapy and radiotherapy-induced oral mucositis. Braz. Dent. J., 21(3):186-92, 2010.

Mínguez, A. \& de Andrés, J. La formulación magistral en la escalera analgésica de la OMS como estrategia de la atención farmacéutica. Rev. Soc. Esp. Dolor, 12(4):23441, 2005.

Molina Giraldo, S. M. \& Estupiñán Guzmán, J. R. Mucositis oral en el paciente con terapia antineoplásica. Med. U. P. B., 29(2):135-43, 2010.

Nicolatou-Galitis, O.; Sarri, T.; Bowen, J.; Di Palma, M.; Kouloulias, V. E.; Niscola, P.; Riesenbeck, D.; Stokman, M.; Tissing, W.; Yeoh, E.; Elad, S.; Lalla, R. V. \& Mucositis Study Group of the Multinational Association of Supportive Care in Cancer/International Society of Oral Oncology (MASCC/ISOO). Systematic review of antiinflammatory agents for the management of oral mucositis in cancer patients. Support Care Cancer, 21(11):3179-89, 2013.

Oberoi, S.; Zamperlini-Netto, G.; Beyene, J.; Treister, N. S. \& Sung, L. Effect of prophylactic low level laser therapy on oral mucositis: a systematic review and meta-analysis. Plos One, 9(9):e107418, 2014.

Peterson, D. E.; Ohrn, K.; Bowen, J.; Fliedner, M.; Lees, J.; Loprinzi, C.; Mori, T.; Osaguona, A.; Weikel, D. S.; Elad, S.; Lalla, R. V. \& Mucositis Study Group of the Multinational Association of Supportive Care in Cancer/ International Society of Oral Oncology (MASCC/ISOO). Systematic review of oral cryotherapy for management of oral mucositis caused by cancer therapy. Support Care Cancer, 21(1):327-32, 2013.

Puyal, M.; Jiménez, C.; Chimenos, E.; López, J. \& Juliá, A. Protocolo de estudio y tratamiento de la Mucositis bucal en los pacientes con hemopatías malignas. Med. Oral, 8:10-8, 2003.

Roopashri, G.; Jayanthi, K. \& Guruprasad, R. Efficacy of benzydamine hydrochloride, chlorhexidine, and povidone iodine in the treatment of oral mucositis among patients undergoing radiotherapy in head and neck malignancies: A drug trail. Contemp. Clin. Dent., 2(1):8-12, 2011.

Sahebjamee, M.; Mansourian, A.; Mohammad, M. H.; Zadeh, M. T.; Bekhradi, R.; Kazemian, A.; Manifar, S.; Ashnagar, S. \& Doroudgar, K. Comparative Efficacy of Aloe vera and Benzydamine Mouthwashes on Radiation-induced Oral Mucositis: A Triple-blind, Randomised, Controlled Clinical Trial. Oral Health Prev. Dent., 13(4):309-15, 2015. 
CUEVAS-GONZÁLEZ, M. V.; ECHEVARRÍA-Y- PÉREZ, E.; DÍAZ-AGUIRRE. C. M. \& CUEVAS-GonZÁLEZ, J. C. Tratamiento de la mucositis oral en pacientes oncológicos. Revisión de la literatura y experiencia en el Hospital General de México. Int. J. Odontostomat., 9(2):289-294, 2015.

Sheibani, K. M.; Mafi, A. R.; Moghaddam, S.; Taslimi, F.; Amiran, A. \& Ameri, A. Efficacy of benzydamine oral rinse in prevention and management of radiation-induced oral mucositis: A double-blind placebo-controlled randomized clinical trial. Asia Pac. J. Clin. Oncol., 11(1):22-7, 2015.

Sonis, S. T. Efficacy of palifermin (keratinocyte growth factor-1) in the amelioration of oral mucositis. Core Evid., 4:199-205, 2010.

Svanberg, A.; Ohrn, K. \& Birgegård, G. Oral cryotherapy reduces mucositis and improves nutrition - a randomised controlled trial. J. Clin. Nurs., 19(15-16):2146-51, 2010.

Tejada Domínguez, F. J. \& Ruiz Domínguez, M. R. Mucositis oral: decisiones sobre el cuidado bucal en pacientes sometidos a radioterapia y quimioterapia conforme a la evidencia. Enferm. Glob., 9(1):1-22, 2010.

Wu, H. G.; Song, S. Y.; Kim, Y. S.; Oh, Y. T.; Lee, C. G.; Keum, K. C.; Ahn, Y. C. \& Lee, S. W. Therapeutic effect of recombinant human epidermal growth factor (RhEGF) on mucositis in patients undergoing radiotherapy, with or without chemotherapy, for head and neck cancer: a double-blind placebo-controlled prospective phase 2 multi-institutional clinical trial. Cancer, 115(16):3699-708, 2009.

Yarom, N.; Ariyawardana, A.; Hovan, A.; Barasch, A.; Jarvis, V.; Jensen, S. B.; Zadik, Y.; Elad, S.; Bowen, J.; Lalla, R. V. \& Mucositis Study Group of the Multinational Association of Supportive Care in Cancer/International Society of Oral Oncology (MASCC/ISOO). Systematic review of natural agents for the management of oral mucositis in cancer patients. Support Care Cancer, 21(11):3209-21, 2013.
Dirección para Correspondencia:

Dr. Juan Carlos Cuevas González

Posgrado e Investigación

Facultad de Odontología

Universidad Juárez del Estado de Durango

AV. Predio Canoas S/N Colonia los Ángeles

C.P 34000, Durango

MÉXICO

Email: cuevas_gonzalez@hotmail.com

Recibido: 30-04-2015

Aceptado: 02-06-2015 